\title{
BEST: a web server for brain expression Spatio-temporal pattern analysis
}

Liyuan Guo ${ }^{1,2}$, Wei Lin ${ }^{1,2}$, Yidan Zhang ${ }^{1,2}$, Wenhan $\mathrm{Li}^{3}$ and Jing Wang ${ }^{1,2^{*}}$ (1)

\begin{abstract}
Background: Dysregulated gene expression patterns have been reported in several mental disorders. Limited by the difficulty of obtaining samples, psychiatric molecular mechanism research still relies heavily on clues from genetics studies. By using reference data from brain expression studies, multiple types of comprehensive gene expression pattern analysis have been performed on psychiatric genetic results. These systems-level spatial-temporal expression pattern analyses provided evidence on specific brain regions, developmental stages and molecular pathways that are possibly involved in psychiatric pathophysiology. At present, there is no online tool for such systematic analysis, which hinders the applications of analysis by non-informatics researchers such as experimental biologists and clinical molecular biologists.

Results: We developed the BEST web server to support Brain Expression Spatio-Temporal pattern analysis. There are three highlighted features of BEST: 1) visualization: it generates user-friendly visual results that are easy to interpret, including heatmaps, Venn diagrams, gene co-expression networks and cluster-based Manhattan gene plots; these results illustrate the complex spatio-temporal expression patterns, including expression quantification and correlation between genes; 2 ) integration: it provides comprehensive human brain spatio-temporal expression patterns by integrating data from currently available databases; 3 ) multi-dimensionality: it analyses input genes as both a whole set and several subsets (clusters) which are enriched according to co-expression patterns, and it also presents the correlation between genetic and expression data.

Conclusions: To the best of our knowledge, BEST is the first data tool to support comprehensive human brain spatial-temporal expression pattern analysis. It helps to bridge disease-related genetic studies and mechanism studies, provides clues for key gene and molecular system identification, and supports the analysis of disease sensitive brain region and age stages. BEST is freely available at http://best.psych.ac.cn.
\end{abstract}

Keywords: Web server, Brain expression pattern analysis, Spatio-temporal, Co-expression cluster, Visualization

\section{Background}

The well-regulated gene expression in the human brain, the highly heterogeneous and lifelong changing organ, is the molecular basis of normal cognitive and behavioural functions. The dysregulated gene expression patterns in brain have been reported in several mental disorders, such as schizophrenia [1], bipolar disorder [2] and Alzheimer's disease [3]. Limited by the difficulty of obtaining samples, although excellent epigenetic studies such as the series of

\footnotetext{
* Correspondence: wangjing@psych.ac.cn

${ }^{1}$ CAS Key Laboratory of Mental Health, Institute of Psychology, Chinese Academy of Sciences, 16 Lincui Road, Chaoyang District, Beijing 100101, China

${ }^{2}$ Department of Psycholog, University of the Chinese Academy of Sciences, Beijing 100049, China

Full list of author information is available at the end of the article
}

works from the psychENCODE project have been reported $[4,5]$, psychiatry molecular mechanism research still relies heavily on clues from genetics studies.

Currently, several data tools provide abundant RNA data from human brains to support gene expression analysis. The Genotype-Tissue Expression (GTEx) (https:// gtexportal.org) provides comparative results of gene expression quantifications in different tissues include human brain [6]; the Expression Atlas (https://www.ebi.ac. uk/gxa/home) provides gene expression quantifications across species and biological conditions [7]. Specific to the human brain, the Allen Brain Atlas (http://human. brain-map.org/) and BRAINSPAN (http://www.brainspan.org/) support heatmap generation to view the gene expression quantifications in multiple brain regions from 
different donors [8-10]. The Human Brain Transcriptome (HBT, http://hbatlas.org/) provides genome-wide, exon-level transcriptome data generated from both hemispheres of postmortem human brains, and its "Developmental Trajectories" module provides principal component analysis of the expression of grouped genes in six brain regions $[11,12]$.

By using reference data from the above resources, multiple types of comprehensive gene expression pattern analysis have been performed on psychiatric genetic results [13-16]. In such studies, the expression statuses of interest genes were reviewed in different brain regions (spatial pattern) and age stages (temporal pattern); the potential functional combinations of genes of interest were presented by their enrichment status in coexpression gene clusters, which were generated by Weighted Correlation Network Analysis (WGCNA) [17]. The analysis results can be shown as intuitive graphics, such as expression heatmaps or enrichment heatmaps in a spatial-temporal matrix, a co-expression network constructed by interest genes. These systems-level spatialtemporal expression pattern analyses integrated genetic data with gene expression data of normal adult brains and identified specific brain regions, developmental stages and molecular pathways that are possibly involved in psychiatric pathophysiology. They also provided valuable evidence for further biological and clinical molecular research.

At present, there is no online tool for such systematic analysis, which hinders the applications of analysis among non-informatics researchers, such as experimental biologists and clinical molecular biologists. Thus, we developed the BEST web server to support Brain Expression Spatio-Temporal pattern analysis. This web tool performs gene expression pattern analysis with reference to pre-integrated spatial-temporal expression data generated from healthy human brains. The analysis results are presented as heatmaps, Manhattan plots, networks, and other types of graphics. A user-friendly user interface module was designed for input data submission, browsing results and download.

\section{Implementation \\ Reference data}

Human single nucleotide polymorphisms (SNPs), genes and brain spatio-temporal expression datasets

rsID and genomic location information of genomewide human single nucleotide polymorphisms (SNPs) were obtained from the NCBI dbSNP database (https://www.ncbi.nlm.nih.gov/SNP/, build 151) [18] on the GRCh38 coordinate. Hugo gene nomenclature committee(HGNC) official symbols and genomic locations of genome-wide human genes (both coding genes and non-coding genes) were downloaded from the Ensembl database (www.ensembl.org), assembly GRCh38.p12 [19]. Eight human brain expression datasets were obtained from BrainSpan Atlas [10, 12, 20, 21], Allen brainmap [8], GTEx [6, 22], and other sources [23-26]. As shown in Fig. 1, samples in expression datasets were categorized based on the corresponding brain region and the age of individual from which it was obtained. In each dataset, the average expression quantification of each gene was calculated based on data from all samples in the same spatio-temporal category. The detailed information of reference expression data and the spatio-temporal categories are described in Additional files 1, 2, 3 and 4. In addition to expression data for brain regions, cell-type-specific expression profiles, which provide specific expression gene sets for astrocytes, endothelial cells, microglia, neurons, and oligodendrocytes, were also used in BEST [27].

\section{Co-expression modules}

Weighted gene correlation network analysis (WGCNA) was performed for five of the eight expression datasets to identify clusters of co-expressed genes [17]. According to the guidelines of the WGCNA R package (Version 1.64-1, http://horvath.genetics.ucla.edu/html/CoexpressionNetwork/Rpackages/WGCNA/), three expression datasets with fewer than eight spatio-temporal categories have not been used in WGCNA analysis. The "blockwiseModules" function in WGCNA was used to construct co-expression networks and detect clusters, and the gene cluster size was maintained between 30 and 5000. The detected co-expression clusters (gene sets and the correlation weights between genes) were stored for subsequent analysis. The statistics of co-expression clusters are shown in Additional files 1, 2, 3 and 4.

\section{Analysis and statistics \\ SNP mapping and gene-based $P$ value calculation}

BEST accepts the human gene list and SNP list (with/ without $P$ value) as input. As shown in Fig. 1, inputs of SNPs are mapped to genes according to their chromosomal locations, and the gene-based $P$ value is computed with an F-test based on a multiple linear principal components regression module by using the MAGMA software [28]; the test is corrected by using a user selected method. Four correction methods are provided, including Bonferroni, Sidak, false discovery rate (FDR)-Benjamini Hochberg (BH), and FDR-Benjamini Yekutili (BY). Linkage disequilibrium (LD) between SNPs is fully accounted for during the computation (28), and LD information for five populations, including African (AFR), mixed American (AMR), East Asian (ESA), European (EUR) and South Asian (SAS), was compiled from Phase 3 of the 1000 genomes project [29]. 


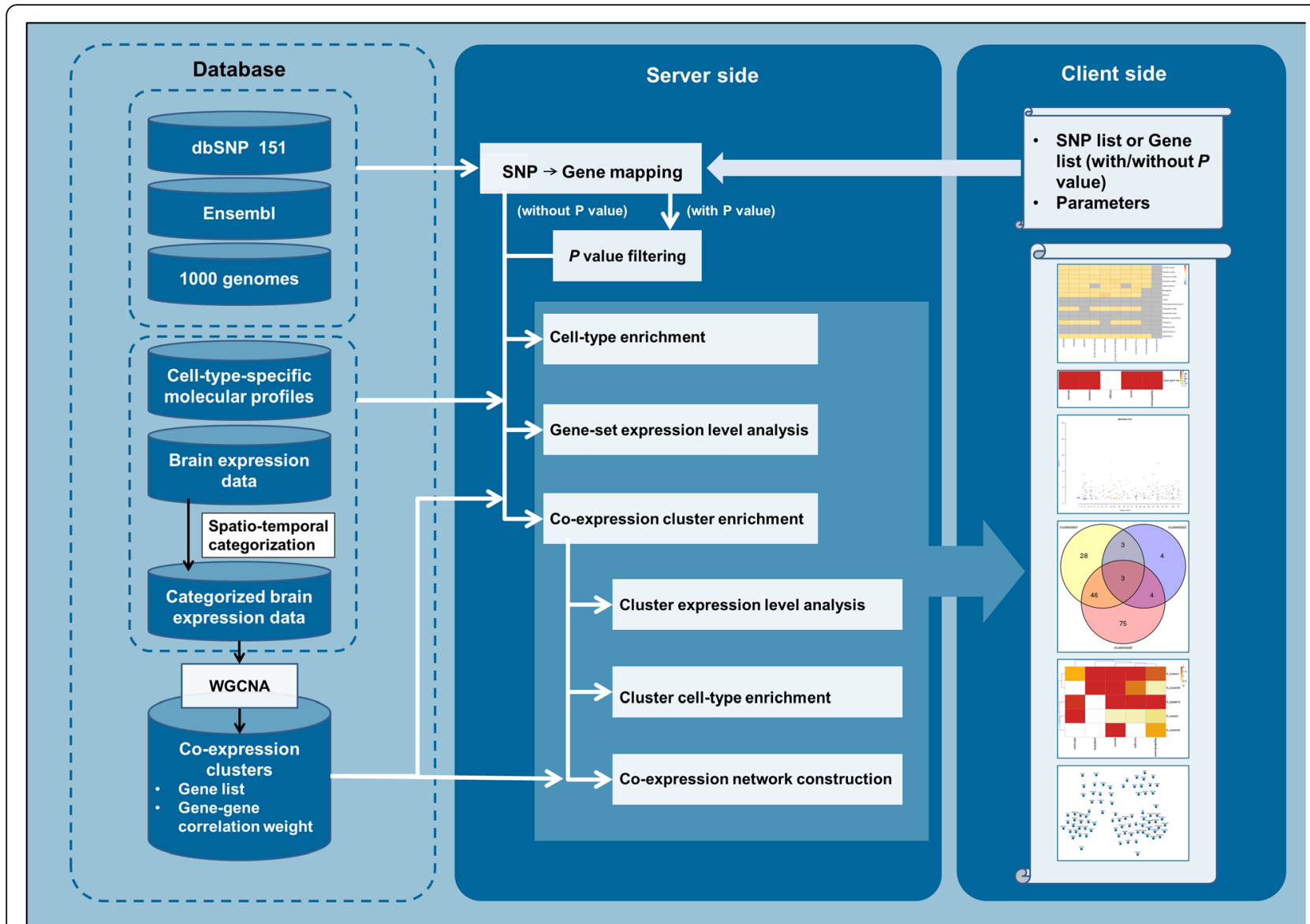

Fig. 1 The system architecture and overview of BEST web server

\section{Gene set-based brain spatio-temporal expression analysis} Input or mapped genes with $P$ values are filtered before expression analysis: genes mapped from inputs of the SNP list with $P$ value are filtered by user selected cutoffs for adjusted $P$ values; inputs of the gene list with $P$ value are filtered by the cutoff of 0.05 . Filtered genes are treated as a whole set, and the average expression quantifications are calculated with reference to user selected expression datasets. Inputs or mapped genes without $P$ values are analysed without any filter. Expression heatmaps are generated according to the analysis result in a matrix of brain regions by age periods (the spatiotemporal matrix). Except for brain region and age period related spatio-temporal analysis, cell-type-specific gene set enrichment is also performed by using Fisher's exact test with the cutoff of 0.05 , and an enrichment heatmap is generated accordingly.

\section{Co-expression gene cluster enrichment and co-expression network construction}

The input or mapped genes are compared with genes in co-expression clusters. Similar to expression analysis, if data were input with $P$ values, the gene set will be filtered before analysis. Specifically, for inputs with $P$ values, gene Manhattan plots are generated by using coexpression clusters as $\mathrm{x}$-axis. Co-expression gene cluster enrichment analysis is performed on the input gene set by using Fisher's exact test with user selected correction method and cutoff, and the enrichment heatmap is generated accordingly. The enrichment results may differ when using co-expression clusters generated from different expression datasets, and so a Venn diagram is calculated to present the numbers of significantly enriched genes by using different reference data. The average expression quantifications of genes in enriched clusters are calculated and presented as expression heatmaps. The connectivity of input/mapped genes in enriched clusters are counted, and the top 20 genes are treated as core genes. Core genes in the top 5 enriched clusters of each dataset are collected into one network file to construct editable co-expression network graphics.

\section{Implementation}

BEST was developed using a server-client design. The server side was implemented using gunicorn (https:// gunicorn.org/), the back-end computing is performed by 
using Python (version v3.6) and images are generated by using R (version v3.3.2). The client side was built based on the React framework (https://reactjs.org/) and JavaScript libraries Bootstrap (https://getbootstrap.com/) and jQuery (version v3.3.1) (https://jquery.com/). The Python framework flask (http://flask.pocoo.org/) was used to launch the server. All reference data and job information are saved in a MySQL database. The graphical network output is enabled with the plugin Cytoscape (version v3.3.2) [30]. Downloadable results of heatmaps, Venn diagrams and plot diagrams are provided in PNG format. BEST has been tested in most major web browsers such as Chrome, Firefox, and Safari.

\section{Results}

BEST provides a user-friendly user interface. After input data upload and parameter selection, a job can be simply submitted. A job is often finished in several minutes, and the results page will be accessed automatically; however, a user can also retrieve the result later by visiting the results URL link that is generated after job submission. A case example is shown in the "A demo run" module to demonstrate usage.

\section{Input and parameters}

BEST accepts the human gene list and SNP list (with/ without $P$ value) as input. Genes should be entered with their official symbol. SNP could be entered with their rs ID or in VCF-like and plink-like format. The detail information of input format is shown in "Documents > Tutorial" page. Three types of mapping rules, LDcorrelation information of five populations and four types of correction methods could be selected in the SNP to gene mapping. Eight human brain expression data resources could be selected as reference data. Four types of correction methods could be selected the coexpression cluster enrichment too..

\section{Output}

As shown in Fig. 2, BEST provides heatmaps, Manhattan plots, Venn diagrams, and networks to illustrate the brain spatio-temporal expression patterns of input.

\section{Expression heatmap of all inputs}

As shown in Fig. 2a, the average expression quantifications of input in all spatio-temporal categories are presented in heatmap form. Although the spatial-temporal categories of each reference dataset are different, BEST generates heatmaps of the same structure (a matrix of 16 brain regions by 10 age stages) to facilitate the comparison between results with reference to different datasets. Specifically, for analysis with reference to RNA-Seq data from Brainspan (dataset 1) and microarray data from the Allen Brain Atlas (dataset 2), heatmaps in the form of matrices of 25 brain regions by 9 age stages and 52 brain regions by 2 age stages are additionally provided, respectively.

\section{Cluster-based Manhattan plots and cluster enrichment heatmap}

As shown in Fig. 2b, the distribution of inputs in coexpression clusters is presented with a gene-based Manhattan plot, which uses co-expression clusters as $\mathrm{x}$-axis and the negative logarithm of gene-based $P$ values as $y$ axis. The enrichment status of inputs in co-expression clusters is presented as a one row enrichment heatmap.

\section{Enriched gene Venn diagram and cell-type enrichment heatmap}

The numbers of significantly enriched genes obtained by using different reference datasets are compared in a Venn diagram, as shown in Fig. 2c. It should be noted that the Venn diagram only presents the statistics of results in reference to spatial-temporal categories in a matrix of the 16 brain regions by 10 age stages. The cell-type specific analysis results of all enriched clusters are shown in enrichment heatmaps, with one cluster for each row.

\section{Expression heatmap of enriched clusters}

Except for average expression quantification of all inputs, BEST calculates the average expression quantifications of genes in each enriched cluster and presents them in expression heatmap form. As shown in Fig. 2d, different gene clusters enriched by the same input gene set may present different spatial-temporal expression patterns.

\section{Core co-expression network of enriched genes}

The enriched clusters in each dataset are prioritized by corrected $P$ values. As shown in Fig. 2e, the core coexpression network of a maximum of the top 5 enriched clusters (if they exist) for each dataset is present as an editable graphic. The core network is constructed by at most 10 input genes (if they exist) with the highest connectivity in each cluster. Moreover, the network files containing co-expression relationships of all input genes in all enriched clusters could be found in the downloaded results folder.

\section{Usage example}

In the "A demo run" page, we provided a usage example by using the best $10 \mathrm{k}$ SNPs (identified by the original study) of a genome-wide association study (GWAS) of major depressive disorder (MDD) as input [31]. As shown in Fig. 3, the demo data was uploaded with an rs ID (with $P$-value) format. The mapping rule "within gene" is selected for SNP gene mapping; the LD between SNPs is accounted for according to reference data from European populations in gene-based $\mathrm{P}$ value calculation. 

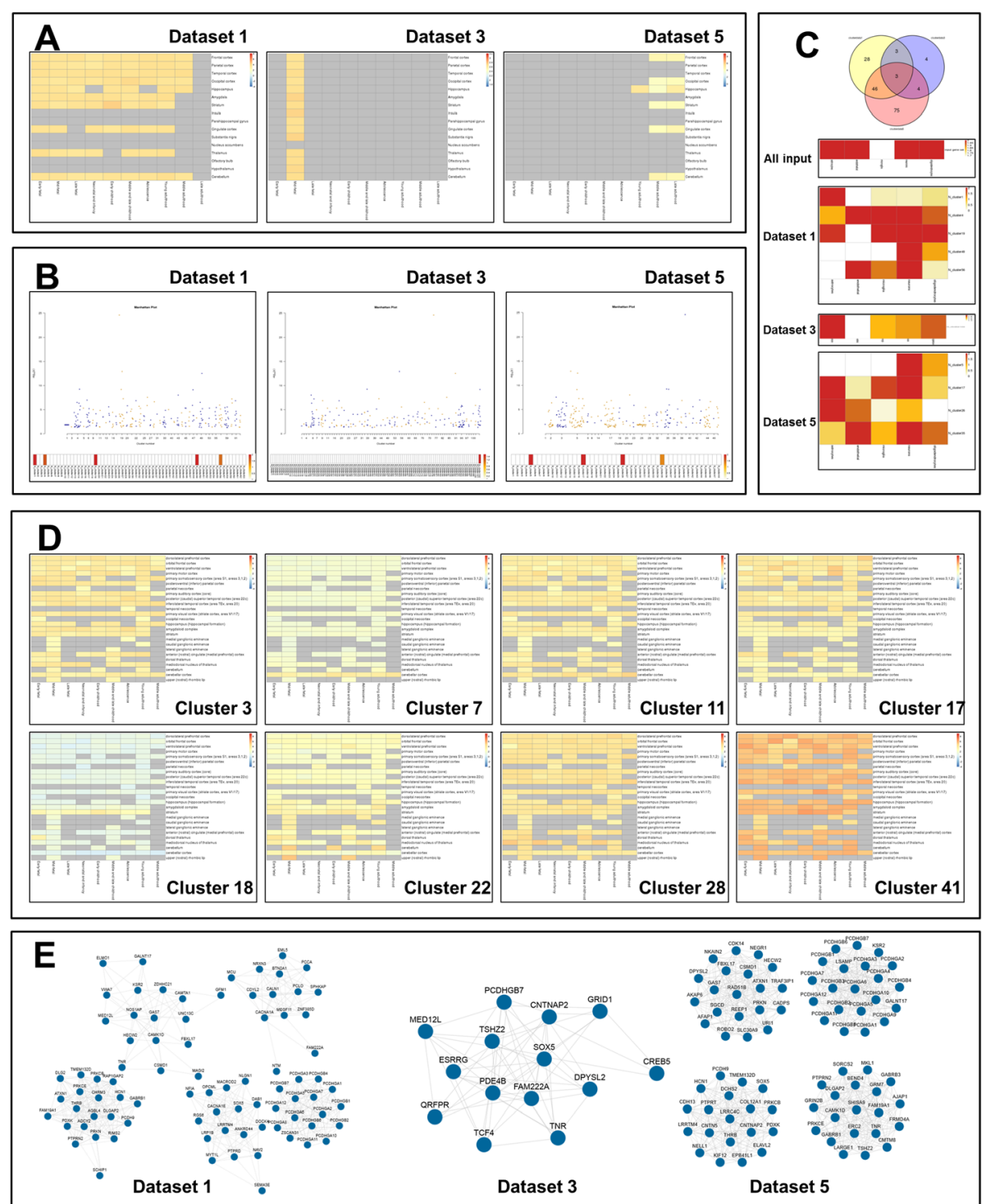

Fig. 2 Selected results of the demo run. a: Expression heatmap of all inputs; $\mathbf{b}$ : Cluster-based Manhattan plots and cluster enrichment heatmap; $\mathbf{c}$ Enriched gene Venn diagram and cell-type enrichment heatmap; $\mathbf{d}$ : Expression heatmap of enriched clusters; e: Core co-expression network of enriched genes

The gene-based $\mathrm{P}$ value is further corrected by Bonferroni with the cut off 0.05. Expression dataset 1 (RNA-Seq data from Brainspan), dataset 3 (microarray data from Allen Brain Atlas) and dataset 5 (RNA-Seq data from Xu C et al., 2018) are selected for expression pattern analysis. All of these datasets include co-expression cluster information, and so the Bonferroni correction method and a cutoff of 0.05 are selected for cluster enrichment.

After analysis, 262 genes were mapped from inputs and the detail gene list can be queried from the "Gene_set.txt" in the downloadable results fold. These genes were enriched respectively in five, one and four co-expression clusters from the three selected data resources in the context of 16 by 10 spatio-temporal matrix. Specific for dataset 1 , these genes were also enriched in eight clusters in the context of 25 by 9 matrix. These enriched coexpression clusters presented complex spatio-temporal expression features. Take results with reference to dataset 1 in 16 by 10 matrix for example, as shown in Fig. 3a, the most significantly enriched cluster is cluster 19 , in which the most significant gene is included. According to the expression heatmap (Fig. 3b), genes in this cluster are expressed in multiple brain regions and a relative upregulation appears in the stage of neonatal to early childhood (in parietal, occipital, and cingulate cortex). Considering the correlation of early life events and adult depression [32], the epigenetics status of genes in this cluster may be involved in the molecular pathogenesis of 


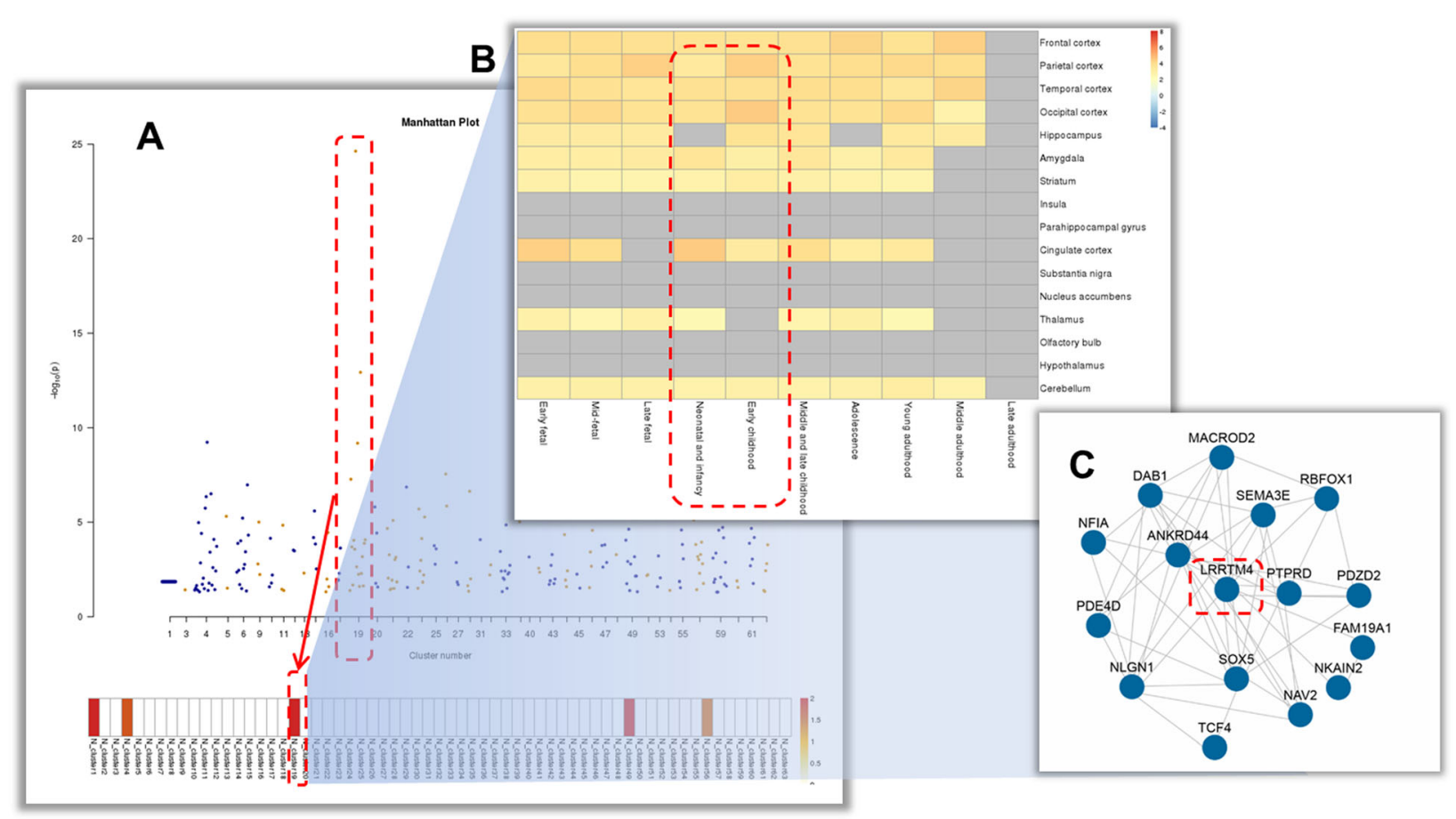

Fig. 3 An usage example of BEST. a: Cluster-based Manhattan gene plots; b: Spatio-temporal expression heatmap; c: Gene co-expression network

MDD. Among the 16 genes enriched in this cluster, the LRRTM4 gene has the most connections in the coexpression network (Fig. 3c). It may play important role in the cluster-related biological processes.

\section{Conclusion}

BEST is a user-friendly web tool to provide gene expression spatial-temporal pattern analysis in human brain. By using this data tool, users can perform a comprehensive analysis on the results of genetic study (such as GWAS or next-generation sequencing based study); users can also obtain an expression pattern of any genes of interest or SNPs which may have accumulated from previous mechanistic studies or animal models.

In BEST, several human brain expression datasets were compiled and processed as reference data of expression pattern analysis. Gene expression profiling is categorized according to sample brain region and age period. Matrices of expression patterns in the brain regions by age periods will provide more detailed clues on gene function. The cell type specification of gene expression is also considered in BEST, which will further expand the understanding of input-related molecular processes.

Differently from most previous tools, BEST does not focus on the expression status of a single gene: it analyses inputs in a more systematic and comprehensive manner. While BEST treats input as a complete gene set, it also categorizes input into several gene subsets according to the gene expression correlation in the reference dataset. Expression spatial-temporal patterns of such subsets will provide richer and more detailed information, and expression characteristics that are offset by the up- and downregulation in the whole set analysis will be more fully demonstrated. The expression correlations within subsets are presented as a network which will reflect the relationship between analysed genes and intuitively demonstrate the potential importance.

All results in BEST are provided as downloadable graphics, and the data by which the graphics were generated, such as the network text files with a Cytoscape applicable format, are also provided. These result figures are easy to interpret; each graphic illustrates an independent question, and the combination of graphics provides more comprehensive and logical interpretation.

To the best of our knowledge, BEST is the first data tool to support comprehensive human brain spatialtemporal expression pattern analysis; it will facilitate a wide range of human brain related studies. BEST helps to bridge disease-related genetic studies and mechanism studies, provides clues for key gene and molecular system identification, and supports the analysis of disease sensitive brain region and age stages. The analysis results of BEST will further provide evidence for clinical molecular studies related to brain diseases, such as disease biomarker identification and drug development. To provide better supports, BEST will be reviewed annually for 
possible update. Besides of including new published brain expression profiling as reference data, BEST will attempts to integrate more types of brain functional data, such as including gene expression coupled structural covariance network [33], when relative methods were developed.

\section{Availability and requirements Project name: BEST \\ Project home page: http://best.psych.ac.cn \\ Operating system: Platform independent \\ Programming language: Python \\ Other requierments: none}

License: Freely available to academic researchers.

Source code available upon request

Any restrictions to use by non-academics: BEST use is restricted to academic and non-profit users

\section{Supplementary information}

Supplementary information accompanies this paper at https://doi.org/10 1186/s12859-019-3222-6.

Additional file 1: Table S1. The summary of reference expression data. Table S2. The age periods in BEST. Table S6. The statistics of COexpression clusters in different datasets

Additional file 2: Table S3. The Summary of Spatio-Temporal categories in reference datasets.

Additional file 3: Table S4. The addational Spatio-Temporal categories in reference dataset 1 .

Additional file 4: Table S5. The addational Spatio-Temporal categories in reference dataset 2

\section{Abbreviations}

AFR: African; AMR: American; BH: Benjamini Hochberg; BY: FDR-Benjamini Yekutili; ESA: East Asian; EUR: European; FDR: False discovery rate; GTEx: Genotype-tissue expression; GWAS: Genome-wide association study; HBT: Human Brain Transcriptome; HGNC: Hugo gene nomenclature committee; LD: Linkage disequilibrium; MDD: Major depressive disorder SAS: South Asian; SNP: Single nucleotide polymorphisms; WGCNA: Weighted correlation network analysis

\section{Acknowledgements}

Not applicable.

\section{Authors' contributions}

JW designed the analysis pipeline. LG designed the user interface. LG and $W L$ implemented the pipeline. $L G$ also drafted the manuscript. $W L$ and $Y Z$ prepared the reference data. WL tested the platform and draft documents in platform. All authors have read and approved the final manuscript.

\section{Funding}

This work was supported by grants from the National Basic Research (973) Program (2015CB351702), CAS Key Laboratory of Mental Health, Institute of Psychology; CAS/SAFEA International Partnership Program for Creative Research Teams [Y2CX131003]; Knowledge Innovation Program of the Chinese Academy of Sciences [KSCX2-EW-J-8].

All funding bodies didn't play any roles in the design of the study and collection, analysis, and interpretation of data and in writing the manuscript.

\section{Availability of data and materials}

Source code of BEST is available on GitHub (https://github.com/GuoLiyuangithub/BEST).
All data generated or analysed during this study are included in this published article [and its supplementary information files].

Ethics approval and consent to participate

Not applicable.

Consent for publication

Not applicable.

\section{Competing interests}

The authors declare that they have no competing interests.

\section{Author details}

${ }^{1}$ CAS Key Laboratory of Mental Health, Institute of Psychology, Chinese Academy of Sciences, 16 Lincui Road, Chaoyang District, Beijing 100101, China. ${ }^{2}$ Department of Psycholog, University of the Chinese Academy of Sciences, Beijing 100049, China. ${ }^{3}$ Oumeng V medical Laboratory, Hangzhou 310013, Zhejiang, China.

Received: 9 August 2019 Accepted: 13 November 2019

Published online: 05 December 2019

References

1. Mimmack ML, Ryan M, Baba H, Navarro-Ruiz J, Iritani S, Faull RL, McKenna PJ, Jones PB, Arai H, Starkey M, et al. Gene expression analysis in schizophrenia: reproducible up-regulation of several members of the apolipoprotein $L$ family located in a high-susceptibility locus for schizophrenia on chromosome 22. Proc Natl Acad Sci U S A. 2002;99(7):4680-5.

2. Chen H, Wang N, Zhao X, Ross CA, O'Shea KS, McInnis MG. Gene expression alterations in bipolar disorder postmortem brains. Bipolar Disord. 2013;15(2): 177-87.

3. Liang WS, Reiman EM, Valla J, Dunckley T, Beach TG, Grover A, Niedzielko $T L$, Schneider LE, Mastroeni D, Caselli R, et al. Alzheimer's disease is associated with reduced expression of energy metabolism genes in posterior cingulate neurons. Proc Natl Acad Sci U S A. 2008;105(11):4441-6.

4. Li M, Santpere G, Imamura Kawasawa Y, Evgrafov OV, Gulden FO, Pochareddy S, Sunkin SM, Li Z, Shin Y, Zhu Y, et al. Integrative functional genomic analysis of human brain development and neuropsychiatric risks. Science. 2018;362(6420). https://science.sciencemag.org/content/362/6420/ eaat7615.long.

5. Gandal MJ, Zhang P, Hadjimichael E, Walker RL, Chen C, Liu S, Won H, van Bakel $H$, Varghese $M$, Wang $Y$, et al. Transcriptome-wide isoform-level dysregulation in ASD, schizophrenia, and bipolar disorder. Science. 2018;362(6420). https://science. sciencemag.org/content/362/6420/eaat8127.long.

6. e GP: Enhancing GTEx by bridging the gaps between genotype, gene expression, and disease. Nat Genet 2017, 49(12):1664-1670.

7. Papatheodorou I, Fonseca NA, Keays M, Tang YA, Barrera E, Bazant W, Burke M, Fullgrabe A, Fuentes AM, George N, et al. Expression atlas: gene and protein expression across multiple studies and organisms. Nucleic Acids Res. 2018;46(D1):D246-51.

8. Hawrylycz MJ, Lein ES, Guillozet-Bongaarts AL, Shen EH, Ng L, Miller JA, van de Lagemaat LN, Smith KA, Ebbert A, Riley ZL, et al. An anatomically comprehensive atlas of the adult human brain transcriptome. Nature. 2012; 489(7416):391-9.

9. Sunkin SM, Ng L, Lau C, Dolbeare T, Gilbert TL, Thompson CL, Hawrylycz M, Dang C. Allen brain atlas: an integrated spatio-temporal portal for exploring the central nervous system. Nucleic Acids Res. 2013;41(Database issue): D996-D1008.

10. Miller JA, Ding SL, Sunkin SM, Smith KA, Ng L, Szafer A, Ebbert A, Riley ZL, Royall JJ, Aiona K, et al. Transcriptional landscape of the prenatal human brain. Nature. 2014;508(7495):199-206.

11. Pletikos M, Sousa AM, Sedmak G, Meyer KA, Zhu Y, Cheng F, Li M, Kawasawa Yl, Sestan N. Temporal specification and bilaterality of human neocortical topographic gene expression. Neuron. 2014;81(2):321-32.

12. Kang HJ, Kawasawa YI, Cheng F, Zhu Y, Xu X, Li M, Sousa AM, Pletikos M, Meyer KA, Sedmak G, et al. Spatio-temporal transcriptome of the human brain. Nature. 2011:478(7370):483-9.

13. Eising E, Huisman SM, Mahfouz A, Vijfhuizen LS, Anttila V, Winsvold BS, Kurth T, Ikram MA, Freilinger T, Kaprio J, et al. Gene co-expression analysis identifies brain regions and cell types involved in migraine 
pathophysiology: a GWAS-based study using the Allen human brain atlas. Hum Genet. 2016;135(4):425-39.

14. Skene NG, Bryois J, Bakken TE, Breen G, Crowley JJ, Gaspar HA, GiustiRodriguez P, Hodge RD, Miller JA, Munoz-Manchado AB, et al. Genetic identification of brain cell types underlying schizophrenia. Nat Genet. 2018; 50(6):825-33.

15. Johnson MR, Shkura K, Langley SR, Delahaye-Duriez A, Srivastava P, Hill WD, Rackham OJ, Davies G, Harris SE, Moreno-Moral A, et al. Systems genetics identifies a convergent gene network for cognition and neurodevelopmental disease. Nat Neurosci. 2016;19(2):223-32.

16. Gandal MJ, Haney JR, Parikshak NN, Leppa V, Ramaswami G, Hartl C, Schork AJ, Appadurai V, Buil A, Werge TM, et al. Shared molecular neuropathology across major psychiatric disorders parallels polygenic overlap. Science. 2018; 359(6376):693-7.

17. Langfelder P, Horvath S. WGCNA: an R package for weighted correlation network analysis. BMC Bioinformatics. 2008;9:559.

18. Sherry ST, Ward MH, Kholodov M, Baker J, Phan L, Smigielski EM, Sirotkin K. dbSNP: the NCBI database of genetic variation. Nucleic Acids Res. 2001; 29(1):308-11.

19. Cunningham F, Achuthan P, Akanni W, Allen J, Amode MR, Armean IM, Bennett R, Bhai J, Billis K, Boddu S, et al. Ensembl 2019. Nucleic Acids Res. 2019;47(D1):D745-51.

20. Johnson MB, Kawasawa YI, Mason CE, Krsnik Z, Coppola G, Bogdanovic D, Geschwind DH, Mane SM, State MW, Sestan N. Functional and evolutionary insights into human brain development through global transcriptome analysis. Neuron. 2009;62(4):494-509.

21. Colantuoni C, Lipska BK, Ye T, Hyde TM, Tao R, Leek JT, Colantuoni EA, Elkahloun AG, Herman MM, Weinberger DR, et al. Temporal dynamics and genetic control of transcription in the human prefrontal cortex. Nature. 2011;478(7370):519-23.

22. Consortium G: Human genomics. The genotype-tissue expression (GTEx) pilot analysis: multitissue gene regulation in humans. Science. 2015; 348(6235):648-60.

23. He Z, Bammann H, Han D, Xie G, Khaitovich P. Conserved expression of lincRNA during human and macaque prefrontal cortex development and maturation. RNA. 2014;20(7):1103-11.

24. Xu C, Li Q, Efimova O, He L, Tatsumoto S, Stepanova V, Oishi T, Udono T, Yamaguchi K, Shigenobu S, et al. Human-specific features of spatial gene expression and regulation in eight brain regions. Genome Res. 2018;28(8):1097-110.

25. He Z, Han D, Efimova O, Guijarro P, Yu Q, Oleksiak A, Jiang S, Anokhin K, Velichkovsky B, Grunewald S, et al. Comprehensive transcriptome analysis of neocortical layers in humans, chimpanzees and macaques. Nat Neurosci. 2017;20(6):886-95

26. Lister R, Mukamel EA, Nery JR, Urich M, Puddifoot CA, Johnson ND, Lucero J, Huang Y, Dwork AJ, Schultz MD, et al. Global epigenomic reconfiguration during mammalian brain development. Science. 2013;341(6146):1237905.

27. Zhang Y, Sloan SA, Clarke LE, Caneda C, Plaza CA, Blumenthal PD, Vogel H, Steinberg GK, Edwards MS, Li G, et al. Purification and characterization of progenitor and mature human astrocytes reveals transcriptional and functional differences with mouse. Neuron. 2016;89(1):37-53.

28. de Leeuw CA, Mooij JM, Heskes T, Posthuma D. MAGMA: generalized geneset analysis of GWAS data. PLoS Comput Biol. 2015;11(4):e1004219.

29. Genomes Project C, Auton A, Brooks LD, Durbin RM, Garrison EP, Kang HM, Korbel JO, Marchini JL, McCarthy S, GA MV, et al. A global reference for human genetic variation. Nature. 2015;526(7571):68-74.

30. Shannon P, Markiel A, Ozier O, Baliga NS, Wang JT, Ramage D, Amin N, Schwikowski B, Ideker T. Cytoscape: a software environment for integrated models of biomolecular interaction networks. Genome Res. 2003;13(11):2498-504.

31. Wray NR, Ripke S, Mattheisen M, Trzaskowski M, Byrne EM, Abdellaoui A, Adams MJ, Agerbo E, Air TM, Andlauer TMF, et al. Genome-wide association analyses identify 44 risk variants and refine the genetic architecture of major depression. Nat Genet. 2018;50(5):668-81.

32. Saleh A, Potter GG, McQuoid DR, Boyd B, Turner R, MacFall JR, Taylor WD. Effects of early life stress on depression, cognitive performance and brain morphology. Psychol Med. 2017:47(1):171-81.

33. Romero-Garcia R, Whitaker KJ, Vasa F, Seidlitz J, Shinn M, Fonagy P, Dolan $\mathrm{RJ}$, Jones PB, Goodyer IM, Consortium N, et al. Structural covariance networks are coupled to expression of genes enriched in supragranular layers of the human cortex. Neuroimage. 2018;171:256-67.

\section{Publisher's Note}

Springer Nature remains neutral with regard to jurisdictional claims in published maps and institutional affiliations.
Ready to submit your research? Choose BMC and benefit from:

- fast, convenient online submission

- thorough peer review by experienced researchers in your field

- rapid publication on acceptance

- support for research data, including large and complex data types

- gold Open Access which fosters wider collaboration and increased citations

- maximum visibility for your research: over $100 \mathrm{M}$ website views per year

At $\mathrm{BMC}$, research is always in progress.

Learn more biomedcentral.com/submissions 